\title{
Metformin and the risk of dialysis
}

The use of metformin as a first-line therapy for patients with type 2 diabetes mellitus (T2DM) is increasing; however, evidence of the risk of kidney problems when metformin is used in these patients has been inconclusive. Now, a nationwide retrospective cohort study from Denmark has demonstrated that metformin is associated with a much higher risk of acute dialysis than that with sulphonylureas.

The study included 168,443 drug-naive patients with T2DM aged $\geq 50$ years who initiated treatment with metformin (119,153 of the patients) or a sulphonylurea between 2000 and 2012. "We calculated absolute 1-year risks of acute dialysis derived from predictions of cause-specific Cox regression models adjusted for potential confounders for acute dialysis, and for death or end-stage renal disease without acute dialysis using a semi-parametric $\mathrm{g}$-formula approach," explains corresponding author Nicholas Carlson.

Within the first year of initiating treatment, 197 patients required acute dialysis, 16 patients developed end-stage renal disease and 7,153 patients died. The analysis revealed that compared with sulphonylureas, metformin was associated with a $50 \%$ increased risk of acute dialysis, which corresponds to an absolute risk difference of 50 per 100,000 . The risk of renal problems was particularly evident in elderly and frail patients. "The results are particularly notable, as the observed absolute risk of acute dialysis in metformin was more than fourfold greater than the absolute risk in the general population, and twofold greater than in patients treated with sulphonylureas," explains Carlson.

"Although the efficacy of metformin in the management of T2DM remains indubitable, our results challenge the perceived renal safety of metformin, particularly in marginal patients," says Carlson. "Specifically, our results underscore the clinical importance of discontinuation of metformin in patients with acute illness, and question the perceived unconditional benefit of metformin as a first-line therapy in elderly patients with renal insufficiency."

Claire Greenhill

ORIGINAL ARTICLE Carlson, N. et al. Metforminassociated risk of acute dialysis in patients with type 2 diabetes: A nationwide cohort study. Diabetes Obes. Metab.http://dx.doi.org/10.1111/ dom.12764 (2016) 